\title{
Médiévales
}

Langues, Textes, Histoire

70 | printemps 2016

Lieux d'hygiène et lieux d'aisance en terre d'Islam (VII $\mathrm{e}_{-}$ $\mathrm{XV}^{\mathrm{e}}$ siècle)

\section{Charlemagne, 814-2014 : bilan historiographique d'une année de commémoration}

Charlemagne, 814-2014: Historiographical Report on a Year of Commemoration

Julian Führer, Marie-Laure Pain et Amélie Sagasser

\section{CpenEdition}

Journals

Édition électronique

URL : http://journals.openedition.org/medievales/7744

DOI : 10.4000/medievales.7744

ISSN : 1777-5892

Éditeur

Presses universitaires de Vincennes

Édition imprimée

Date de publication : 23 juin 2016

Pagination : 251-269

ISSN : 0751-2708

Référence électronique

Julian Führer, Marie-Laure Pain et Amélie Sagasser, « Charlemagne, 814-2014 : bilan historiographique d'une année de commémoration », Médiévales [En ligne], 70 | printemps 2016, mis en ligne le 15 juin 2018, consulté le 21 décembre 2020. URL : http://journals.openedition.org/medievales/7744 ; DOI : https://doi.org/10.4000/medievales.7744 


\section{Charlemagne, 814-2014 : bilan historiographique d'une année de commémoration}

Nous nous proposons ici de dresser un tableau non exhaustif des parutions et des manifestations les plus significatives dans la perspective d'un bilan du mille deux centième anniversaire de la mort de Charlemagne. La recherche sur Charlemagne, son règne, son empire et son époque a-telle connu un renouveau significatif ? Quelles nouvelles approches peuvent être discernées ? Les traditions historiographiques de chaque pays ont-elles influencé les études et les travaux sur le sujet?

Tout d'abord, quel rôle assigne-t-on à l'empire carolingien ? Si l'on s'intéresse aux premiers éléments qui ouvrent certains ouvrages de synthèse, comme les manuels d'Histoire de France, on peut constater qu'ils commencent le plus souvent avec Clovis et non avec Charlemagne. Il est vrai que Charles le Chauve, petit-fils de Charlemagne, est désigné dans la collection des « Chartes et Diplômes » comme « roi de France ${ }^{1}$ ». Ce titre choisi il y a bien longtemps fait donc remonter l'existence d'un royaume de France au IX ${ }^{\mathrm{e}}$ siècle. Mais Charlemagne a longtemps été un personnage que se disputaient la France et l'Allemagne. Il suffit d'évoquer les questions territoriales liées aussi bien à l'histoire de l'empire carolingien qu'à l'histoire des $\mathrm{XIX}^{\mathrm{e}}$ et $\mathrm{XX}^{\mathrm{e}}$ siècles. Ainsi, dans les manuels d'Histoire allemande, Charlemagne tient souvent une place particulière : celui de père fondateur de ce qui devint l'empire germanique des Ottoniens puis, bien des siècles plus tard, l'Allemagne.

Après 1945 et la fin manifeste du Reich, l'historiographie allemande a quelque peu évité le sujet d'un empire sous domination germanique ou allemande et a préféré s'interroger sur l'essence de la nation allemande en

1. Recueil des actes de Charles II le Chauve, roi de France, commencé par A. GIRY, continué par M. Prou puis G. TESSIER, terminé et publié sous la direction de C. BRUNEL, 3 vol., Paris, 1943-1955 (Chartes et diplômes relatifs à l'Histoire de France, 3). 
réinventant ses origines, avec comme terrain de recherche de prédilection le $\mathrm{X}^{\mathrm{e}}$ siècle, marqué par le gouvernement des Ottoniens, considérés comme les premiers rois allemands choisis par des assemblées qui avaient tourné le dos aux Carolingiens ${ }^{2}$. D'ailleurs le royaume des Ottoniens au nord des Alpes ressemblait de près à la délimitation de la République Fédérale d'Allemagne d'avant la réunification de $1990^{3}$. De nos jours, l'empire carolingien semble revenu à la mode. De nouvelles questions ont été posées et de nouvelles méthodes ont été appliquées, surtout dans le domaine de l'étude des manuscrits (dans le sillon de la numérisation massive des fonds de bibliothèques). Il est néanmoins certain que, dans une ou deux générations, nous découvrirons nos propres anachronismes.

\section{Publications en France et en Allemagne}

À l'occasion du mille deux centième anniversaire de la mort de Charlemagne, un certain nombre d'ouvrages ont été publiés. Du côté allemand, le nombre des publications liées aux commémorations a été plus élevé qu'en France : on compte de nouvelles biographies de Charlemagne, un ouvrage sur Éginhard et son rapport avec Charlemagne, deux catalogues d'expositions et, parmi les publications de taille plus restreinte, un article scientifique replaçant l'empereur dans l'histoire globale. Du côté français, on relève essentiellement la publication d'une édition de texte (traduit et commenté), ainsi que les actes d'un colloque et la publication d'une thèse.

\section{Les ouvrages scientifiques}

Parmi les biographies parues en Allemagne, deux s'adressent plutôt à un cercle de lecteurs familiers des études historiques et ont pour but de faire le point sur l'état de la recherche relative au personnage de Charlemagne. Euvres de Johannes Fried et de Stefan Weinfurter, elles sont parues en

2. L'origine de la controverse était la parution du livre de G. TellenBACH, Königtum und Stämme in der Werdezeit des Deutschen Reiches, en 1939. Pour un bref résumé des points essentiels de son argumentation, voir G. TELLENBACH, «Wann ist das deutsche Reich entstanden ? », Deutsches Archiv für Erforschung des Mittelalters, 6 (1943), p. 1-41, repris dans H. KäMPF éd., Die Entstehung des Deutschen Reiches, Darmstadt, 1956, p. 171-212 (Wege der Forschung, 1). La collection «Wege der Forschung », inaugurée en 1956 par ce volume, réunit des réimpressions d'articles ayant marqué le cours de la recherche et compte aujourd'hui environ 700 volumes.

3. Au sujet des usages parfois surprenants de l'histoire de l'empire ottonien dans le contexte de l'histoire de la RFA des années cinquante, voir M. PAPE, «Lechfeldschlacht und NATO-Beitritt. Das Augsburger "Ulrichsjahr" 1955 als Ausdruck der christlichabendländischen Europaidee in der Ära Adenauer », Zeitschrift des Historischen Vereins für Schwaben, 94 (2001), p. 269-308. 
librairie presque au même moment. Elles se révèlent être des contributions majeures.

Le livre de Johannes Fried peut être considéré comme la biographie la plus détaillée du personnage depuis le Charlemagne de Jean Favier paru en $1999^{4}$. En quelque 736 pages, l'auteur retrace la vie du premier empereur médiéval d'Occident en commençant par son enfance et en terminant par deux chapitres portant d'une part sur sa mort et d'autre part sur son mythe et sa mémoire dans les mille deux cents ans qui ont suivi la fin de son règne. Il replace l'empereur dans son contexte historique en décrivant de manière très détaillée son environnement. Il évoque le rôle et l'implication de Charlemagne dans son empire et dans ses relations avec les périphéries : sont abordés l'empereur guerrier et conquérant, les structures politiques et les réformes, la cour et la redéfinition du pouvoir impérial. Il ne s'agit pas d'une biographie « classique » selon une définition moderne du genre - ce que Johannes Fried exprime dès le début de son ouvrage dans son avantpropos et son prologue ${ }^{5}$ :

Ce livre ne constitue pas un roman, mais néanmoins une fiction. Il décrit l'image que l'auteur se fait de Charlemagne. Il est formé et teinté de manière subjective, même si les témoignages ont été consultés. La profondeur d'une vie d'il y a mille deux cents ans ne peut plus être sondée. Ainsi il ne reste plus que la pure imagination.

Ceci est principalement dû à l'état lacunaire des sources. Nous ne possédons presque aucun témoignage de Charlemagne en tant que personne. La recherche historique n'a guère pu tirer d'informations valables sur la personnalité du souverain ni de la Vie écrite par Éginhard ni des autres sources. Johannes Fried doit se servir d'autres méthodes pour s'approcher de son personnage, et c'est pourquoi il parle de fiction. En rassemblant un grand nombre de détails (comme par exemple une énumération du bétail), il essaie de faire comprendre au fil d'une narration captivante le fonctionnement $\mathrm{du}$ monde sur lequel Charlemagne a régné. De cette description résulte l'image d'un Charlemagne en homme de son temps, luttant en tant que premier empereur médiéval d'Occident qui essaie d'agrandir son empire mais aussi de le stabiliser, en l'unifiant tant au niveau séculier qu'au plan ecclésiastique. Il s'agit d'une œuvre très littéraire qui rassemble avec rigueur, dans un seul ouvrage, les « pièces de puzzle » sur le monde carolingien en faisant un point sur l'état de la recherche de cette

4. J. FRIED, Karl der Große. Gewalt und Glaube. Eine Biographie, Munich, 2013 ; J. FAVIER, Charlemagne, Paris, 1999.

5. J. FRIED, Karl der Große..., p. 9. 
période du haut Moyen Âge, même si finalement cette approche n'aboutit pas forcément à de nouveaux résultats.

Stefan Weinfurter a également accepté le défi d'écrire la biographie de ce personnage du haut Moyen Âge, mais de manière plus condensée ${ }^{6}$. Son approche est plus classique. Après une présentation de sa perspective et de l'importance que possède encore cet empereur mille deux cents ans après sa mort, un point sur l'état des sources et un chapitre sur le mythe de Charlemagne, Stefan Weinfurter entame la narration de la vie de l'empereur franc en adoptant un plan chronologique : son enfance, sa rivalité avec son frère, ses guerres, l'organisation du pouvoir, le problème de la succession, sa relation avec les sciences et l'idée de l'unification de la foi, etc. - en terminant par sa mort. Les actions de l'empereur et de son entourage restent au centre de l'ouvrage. L'objectif principal et l'ambition majeure de l'empereur furent ce que Stefan Weinfurter qualifie de Vereindeutigung, de « clarification ». Il entend par ce terme la lutte de l'empereur pour la transparence, la disparition des ambiguïtés, que ce soit en tant qu'homme politique ou en tant qu'homme d'Église, ou simplement en tant qu'homme de son temps. L'Église, son unité et, plus globalement, l'idée de l'unification de la chrétienté restent durant ces transformations au centre de ses actions. Stefan Weinfurter tire ce concept de Vereindeutigung de l'idée d'une Vereinheitlichung, terme que l'on peut traduire par « effort d'unification », voire «d'uniformisation » de l'empire. Selon l'auteur, ce phénomène est limité aux réformes purement séculières, par exemple celles des institutions ou du droit. L'historien de Heidelberg aboutit à la conclusion que Charlemagne menait une politique cohérente en essayant de clarifier toutes ses décisions et actions avec de vastes réformes juridiques, liturgiques et théologiques, culturelles et littéraires, et ce jusqu'aux transformations architecturales. Un exemple de cette volonté de stabilité fut, entre autres, la construction d'un palais à Aix-la-Chapelle comme centre du pouvoir. Cette lutte quasi obsessionnelle vers une Vereindeutigung permanente fut incarnée selon l'auteur par l'empereur en personne vers la fin de sa vie.

Parmi les publications scientifiques germanophones, et afin de mesurer l'importance de Charlemagne pour l'histoire globale (courant de la recherche jeune et actif depuis quelques années), il convient aussi de mentionner un article de Michael Borgolte ${ }^{7}$. Celui-ci pose la question du transfert des idées ainsi que des concepts de réseau et d'interconnectivité au temps de Charlemagne. Peut-on obtenir davantage d'informations sur cette époque avec des approches issues de l'histoire globale ? L'auteur aborde ce sujet en prenant comme exemple la politique d'expansion de l'empereur,

6. S. WEINFURTER, Karl der Große. Der heilige Barbar, Munich, 2013.

7. M. BorgOlTE, « Karl der Große. Sein Platz in der Globalgeschichte », Saeculum, 63 (2013), p. 167-188. 
les migrations et le commerce à distance. Il parvient à la conclusion que l'empire restait purement européen. Dans sa politique d'expansion comme dans sa politique de migration, Charlemagne a principalement cherché l'intégration des chrétiens ou des païens ; en revanche, dans ses guerres d'expansion, il a évité les musulmans. Pour l'époque carolingienne, une interconnectivité existait peut-être seulement au niveau du commerce en Méditerranée. Pour autant, il serait exagéré, d'après Michael Borgolte, de déduire du contact avec les Arabes que Charlemagne a été un personnage d'importance à l'échelle globale.

En marge de ces publications, le livre de Steffen Patzold, dont le titre peut se traduire par Moi et Charlemagne. La vie du courtisan Éginhard ${ }^{8}$, s'approche de son personnage d'une manière originale. L'ouvrage est consacré au biographe de Charlemagne, Éginhard, l'auteur de la Vita Caroli magni. Il s'agit de la première biographie consacrée à ce personnage, ce qui offre un autre angle de vue sur l'empereur. Une lacune dans la recherche - certes due à la difficile interprétation des sources - est ainsi comblée. C'est porté par la curiosité que Steffen Patzold va à la rencontre de ce personnage. Il avoue dès la première ligne son empathie avec son sujet, au point qu'il aurait bien aimé pouvoir prendre un café avec son personnage ; mais au-delà de cette remarque introductive qui peut paraître anecdotique, les notes en fin d'ouvrage témoignent d'une réflexion très poussée sur le personnage et ses œuvres dans les réseaux de son temps. L'auteur situe la rédaction de la Vita Caroli en l'an 829, donc quinze ans après la mort de Charlemagne, d'où un accent particulier de la narration sur les années du règne de Louis le Pieux, fils et successeur de l'empereur. Au premier abord, cela peut paraître contredire le titre du livre. Mais il ne faut pas oublier que le choix d'une focalisation sur un homme très proche de l'empereur permet de déduire un certain nombre d'informations au sujet du fonctionnement de la cour impériale. L'auteur, qui souhaite éveiller l'intérêt et partager sa curiosité avec les lecteurs, essaie de se mettre à la place du personnage et se pose un grand nombre de questions. Il propose de trouver ses réponses en retraçant la vie d'Éginhard à partir d'une étude critique des sources existantes, notamment les poèmes de Théodulfe, la Vita Caroli, le récit fait par Éginhard lui-même de la translation des saints Marcellin et Pierre, ainsi qu'une petite biographie de Walahfrid Strabon, qui a réédité la Vie de Charlemagne. Mais l'historien ne cache pas les limites de son approche. Là où les sources ne sont pas suffisamment fiables, il avance des hypothèses ou ne craint pas de laisser une question sans réponse. L'auteur souligne qu'Éginhard était un marginal à la cour en raison de ses origines modestes et de sa carrière atypique. Il y fut appelé en raison de son esprit vif, car

8. S. PATZOLD, Ich und Karl der Große. Das Leben des Höflings Einhard, Stuttgart, 2013. 
seul celui qui était capable d'interpréter correctement les textes sacrés, seul celui qui savait comment prier Dieu dans un langage adéquat et correct, seul celui-là pouvait espérer ne pas mésentendre la volonté de Dieu ; seul celui-là pouvait espérer obtenir la bienveillance du Tout-Puissant ${ }^{9}$.

\section{Ainsi Steffen Patzold en conclut qu'Éginhard a rédigé}

un texte très politique et personnel à la fois. La Vita de Charlemagne devait l'aider à quitter la cour dans un contexte menaçant, à vivre auprès de ses saints à Mulinheim - tout en restant un homme sage et expérimenté, bien informé et sollicité ${ }^{10}$.

Tout comme la vie que Charlemagne a tenté de mener, sa Vita écrite par Eginhard fut gouvernée par le sentiment religieux et l'effort de mener une vie exemplaire, orientée vers la salvation.

Du côté des publications françaises également, le biographe de Charlemagne et son œuvre ont fait l'objet d'études très poussées, mais sous un angle différent. Un des événements majeurs de cette année 2014 aura été la réédition de la Vie de Charlemagne d'Éginhard sous la direction de Michel Sot et de Christiane Veyrard-Cosme avec une nouvelle traduction française. Cette Vie avait été traduite du latin en 1923 par Louis Halphen mais, bien que la recherche lui doive beaucoup, ce travail était devenu dépassé, tant en raison de la langue employée que de ses interprétations : il convenait donc de revenir, presqu'un siècle plus tard, sur cette traduction. Celle que proposent Michel Sot et Christiane Veyrard-Cosme est entièrement nouvelle et a été associée à des notes désormais essentielles puisque fondées sur les dernières découvertes historiques ${ }^{11}$. Afin de mener cette édition à bien, les auteurs se sont tout particulièrement concentrés sur les modèles stylistiques d'Éginhard: principalement Cicéron, César et Suétone.Ainsi, l'ouvrage offre une lecture inédite du texte carolingien en alliant une démarche historique à une analyse littéraire. Cette Vie, qui est et demeure l'une de nos sources les plus évocatrices sur Charlemagne, est également appréciée, à travers cette nouvelle approche, en tant que document majeur du règne de Louis le Pieux (puisque rédigé à une date incertaine, entre 817 et 836). En donnant plus qu'une simple traduction, les auteurs ont souhaité inscrire cette nouvelle édition dans l'histoire politique et culturelle du règne de Charlemagne : leur travail participe ainsi au renouvellement de nos connaissances sur le sujet.

9. Ibid., p. 296.

10. Ibid., p. 295.

11. Éginhard, Vie de Charlemagne, éd. M. Sot et C. Veyrard-Cosme, Paris, 2014 (Classiques de l'Histoire au Moyen Âge, 53). 
Martin Gravel s'est quant à lui intéressé à la gestion et à la construction de l'empire carolingien dans un livre tiré d'une thèse parisienne ${ }^{12}$. Comme l'indique le titre, l'auteur entend évaluer les distances qui séparent le souverain de ses sujets et celles qu'il doit parcourir. Des limites dont souffrent ces déplacements découle la nécessité d'établir une hiérarchie et des réseaux pour gouverner l'ensemble du vaste royaume. Martin Gravel propose alors une étude des communications et des échanges au temps de Charlemagne et de son fils, Louis le Pieux. Outre une analyse portant sur les aspects techniques et matériels de ces circulations d'hommes et d'objets, l'auteur cherche à démontrer la manière dont s'exerce le pouvoir et dont circulent les informations dans l'empire. Il rattache à juste titre cette notion à celle de l'indispensable omniprésence du souverain sur ses terres, présence qui est illustrée à travers divers symboles. Sont ainsi également étudiés dans cet ouvrage l'exercice du pouvoir par le moyen de réseaux de relations et l'utilisation de symboles représentatifs du souverain - sujets particulièrement en vogue dans l'historiographie actuelle. Même si l'on considère que les Carolingiens avaient un sens de l'ordre très développé, il va de soi que toute communication à distance entraînait une perte de temps et, par là, une distorsion des renseignements relatifs à chaque région. La rapidité avec laquelle on pouvait diffuser des informations est néanmoins frappante. Une bribe d'information que l'auteur considère comme fiable rapporte ainsi que la nouvelle de la mort de Charlemagne à Aix-la-Chapelle est arrivée dans le Limousin seulement six jours plus tard - ce qui nécessitait bien sûr l'existence d'un réseau de relais pour les messagers ${ }^{13}$.

Les capitulaires mentionnent assez souvent des tentatives de mise en place de voies fiables de communication, mais comme toujours nous savons assez peu de choses du degré de réalisation effective de ces circulaires. Un grand projet de réédition des capitulaires sous la direction de Karl Ubl, initié à l'Université de Cologne au cours de cette année 2014, pourra peut-être, lorsqu'il aura abouti, jeter les nouvelles bases d'une étude de ces écrits ${ }^{14}$. Le sous-titre du livre de Martin Gravel - Réaliser l'empire - souligne l'importance des campagnes militaires qui intéressent aussi Bernard Bachrach ${ }^{15}$ et qui, par exemple chez Éginhard, semblent parfois former l'élément central de l'empire carolingien ; ces conquêtes

12. M. GRAVEL, Distances, rencontres, communications : réaliser l'empire sous Charlemagne et Louis le Pieux, Turnhout, 2012.

13. Ibid., p. 87-90 : les cours se déplaçaient à raison de 20 à $35 \mathrm{~km}$ par jour, le cursus publicus des Romains à $75 \mathrm{~km}$ par jour ; en cas d'urgence, on pouvait maîtriser $130 \mathrm{~km}$ par jour (et même $300 \mathrm{~km}$ chez les Romains dans des cas isolés).

14. Capitularia. Edition der fränkischen Herrschererlasse, en ligne : http://capitularia. uni-koeln.de/ (consulté le 2 décembre 2015).

15. B. S. BACHRACH, Charlemagne's Early Campaigns (768-777). A Diplomatic and Military Analysis, Leyde/Boston, 2013 (History of Warfare, 82). 
une fois faites, il fallait organiser le tout et trouver un mode de gestion. François-Louis Ganshof avait déjà relevé, dans un article devenu célèbre, qu'à cet égard les difficultés paraissaient, à l'époque, insurmontables ${ }^{16}$. Les rébellions fréquentes en Saxe, l'opposition intérieure contre les actions de Charlemagne, et finalement la rébellion ouverte contre Louis le Pieux montrent que, même si la légitimité du gouvernement des Carolingiens était certainement incontestée, il n'en était pas de même quant au mode de gouvernement ${ }^{17}$.

Les symboles de la royauté carolingienne ont été au cœur d'une discussion menée lors d'un colloque, tenu en 2008 et intitulé Charlemagne et les objets : des thésaurisations carolingiennes aux constructions mémorielles ${ }^{18}$, dont les actes ont été publiés en 2014. L'idée était de rassembler les objets associés à la mémoire de Charlemagne : trésors d'orfèvrerie, pièces liturgiques ou profanes, éléments de mobilier, manuscrits. Il convenait alors de revenir sur la notion d'objet sous le règne de Charlemagne et sur sa place dans la société de l'époque. De là, la recherche s'est tournée vers la thésaurisation et l'intérêt des premiers Carolingiens pour les inventaires de trésors (d'églises notamment) qui leur permettaient alors de quantifier les richesses du royaume. Mais il s'agissait aussi d'appréhender la célébration de l'empereur à travers des objets qualifiés de mémoriels. Des travaux ont été consacrés à Charles le Chauve et à sa vocation à créer des objets comme au temps de son prédécesseur. La motivation de Charles le Chauve a fait l'objet de nouveaux débats afin de nuancer les propos faisant de lui un simple imitateur. D'autres sujets ont été réévalués, comme la légende du trésor de Conques et de sa constitution. Ici, le chercheur s'intéresse davantage à comprendre l'effet qu'un tel trésor a eu sur la société carolingienne et médiévale. C'est enfin la construction de l'image de Charlemagne grâce aux objets qui a été présentée. Une approche de l'objet lie ainsi histoire et histoire de l'art dans la réalisation d'un même objectif, celui de la compréhension historique du règne de Charlemagne, que l'on pourrait aisément comparer à celle menée par Marie-Pierre Laffitte et Charlotte Denoël dans un ouvrage publié à l'occasion d'une exposition de la Bibliothèque nationale de France en $2007^{19}$. À côté des publications

16. F.-L. GANSHOF, « La fin du règne de Charlemagne - une décomposition ? », Zeitschrift für schweizerische Geschichte, 28 (1948), p. 433-452 ; voir aussi ID., « L'échec de Charlemagne », Comptes rendus des séances de l'Académie des inscriptions et belles-lettres, 91 (1947), p. 248-254.

17. K. BRUNNER, Oppositionelle Gruppen im Karolingerreich, Vienne/Cologne/Graz, 1979.

18. P. CORDEZ éd., Charlemagne et les objets : des thésaurisations carolingiennes aux constructions mémorielles, Berne, 2012.

19. M.-P. LAFFitTE, C. DENOËL éd., Trésors carolingiens. Livres manuscrits de Charlemagne à Charles le Chauve, Paris, 2007. 
germanophones et francophones, on peut également citer un ouvrage de Geoffrey Koziol ${ }^{20}$ qui, plus qu'un travail sur la diplomatique, se présente également comme une étude de la culture mémorielle, de l'histoire des rituels et un essai de psychologie historique. Les souverains carolingiens laissaient des marques de présence et les établissaient dans l'espace par le biais des diplômes émis ici et là pour des destinataires venant parfois de loin.

L'exposition de la Bibliothèque nationale s'est tenue en 2007, le colloque sur les objets, bien que paru en 2014, s'est déroulé en 2008, et le livre de Geoffrey Koziol est paru en 2012. Ces publications sont donc le fruit de travaux et de réflexions entamés depuis plusieurs années. Le regain d'intérêt pour Charlemagne et les Carolingiens n'est pas totalement inédit et propre à l'année 2014 qui, néanmoins, en tant que date commémorative, a ravivé le sujet et a été l'occasion de s'y intéresser davantage.

\section{Les ouvrages de vulgarisation}

On note également un regain d'intérêt pour l'empereur carolingien de la part d'un public non scientifique, notamment Outre-Rhin. On signalera ainsi deux biographies en allemand destinées à un public plus large, un ouvrage suivant un plan de «questions-réponses », et un supplément de l'hebdomadaire Der Spiegel. Les titres des deux livres sont presque identiques et évoquent "Charlemagne, l'empereur (ou le souverain) le plus puissant du Moyen Âge ». Le premier est l'œuvre de la journaliste Karin Schneider-Ferber, qui essaie de se mettre à la place de l'empereur et retrace, sur un ton divertissant et accessible, la vie de son personnage ${ }^{21}:$ des illustrations et des vignettes explicatives complètent le récit chronologique relatant les faits principaux (tous connus) sur Charlemagne. Le second ouvrage $^{22}$ constitue une version enrichie d'un numéro du magazine Geschichte, paraissant sous les auspices de Der Spiegel ${ }^{23}$. Dietmar Pieper et Johannes Saltzwedel ont rassemblé une trentaine de contributions de vingtdeux auteurs, majoritairement des journalistes mais aussi des professeurs d'université, qui illustrent les différentes facettes - les faits demeurant bien connus - par des genres d'essais très divers. On y trouve également une interview de Johannes Fried et des fac-similés de manuscrits avec les

20. G. KozIOL, The Politics of Memory and Identity in Carolingian Royal Diplomas. The West Frankish Kingdom (840-987), Turnhout, 2012.

21. K. SCHNEIDER-FERBER, Karl der Große. Der mächtigste Herrscher des Mittelalters, Darmstadt, 2013.

22. D. PiePer, J. SAltzWedel éd., Karl der Große. Der mächtigste Kaiser des Mittelalters, Munich, 2013.

23. Der Spiegel : Geschichte, J. SALTZWEDEL éd., Karl der Große, der mächtigste Kaiser des Mittelalters, juin 2012. 
traductions en regard et des reproductions d'objets. Ces trois publications (si on tient compte du numéro du Spiegel) avaient pour but de rendre le personnage de Charlemagne accessible à un public intéressé, mais pas obligatoirement spécialiste, afin de servir de guide durant les festivités. Malgré certes quelques lacunes, cela est tout à fait positif.

Un autre type d'ouvrage également destiné à un large public est celui des historiens Martina Hartmann, vice-présidente des Monumenta Germaniae Historica, et Wilfried Hartmann ${ }^{24}$. Tous deux spécialistes de l'époque carolingienne, ils répondent à « 101 questions » sur Charlemagne, classées en onze parties thématiques. Il s'agit pour eux d'illustrer la diversité du monde de l'empereur dans l'empire franc - de l'alimentation et de la sexualité jusqu'à l'organisation du droit et aux relations de l'empire et de son empereur avec l'Église - en rédigeant les notices dans une langue très compréhensible et en expliquant les termes techniques. Malgré la nécessaire simplification, eu égard au public visé, les auteurs ne négligent pas de fonder leurs assertions sur les sources primaires ni de dévoiler les limites et les doutes liés à la transmission lacunaire ou à l'état problématique des textes. Pour faciliter le repérage dans la chronologie des événements, le livre se clôt sur une chronologie, une généalogie de la dynastie carolingienne et un index des noms de lieux et de personnes.

\section{Rencontres scientifiques en France et en Allemagne}

Outre ces publications, la célébration de la mort de Charlemagne a été l'occasion pour les chercheurs d'organiser colloques et tables rondes. L'Institut historique allemand de Paris a ainsi choisi de réunir des spécialistes du règne de Charlemagne lors du colloque international «Charlemagne : les temps, les espaces, les hommes. Construction et déconstruction d'un règne », en mars 2014. Il s'agissait d'explorer de nouveaux horizons sur le règne de ce monarque et de l'appréhender dans une perspective pluridisciplinaire ${ }^{25}$. En premier lieu, il convenait évidemment de commémorer le « père de l'Europe » et fondateur de l'empire, mais ce colloque se devait de renouveler nos connaissances sur le sujet et de revenir ainsi sur la part des traditions et innovations qui caractérisèrent son règne. Pour cela, il a fallu se détacher de la tendance historiographique attribuant à Charlemagne des réalisations qui, finalement, se sont révélées être des emprunts à une époque antérieure

24. M. et W. Hartmann, Die 101 wichtigsten Fragen - Karl der Große und seine Zeit, Munich, 2014.

25. Le carnet scientifique http://charlemagne.hypotheses.org/ permet d'accéder à des vidéos de toutes les interventions du colloque ; voir aussi le compte rendu (en allemand) du colloque par A. SAGASSER, paru en ligne le 25 juin 2014 (http://www.hsozkult.de/ conferencereport/id/tagungsberichte-5434). 
ou le résultat d'expériences diverses, parfois élaborées aux périphéries de l'empire. Ainsi, les recherches se sont focalisées sur ces périphéries du royaume qui ont constitué des laboratoires d'innovations. Dans ce colloque, dont l'intitulé reprend les termes « construction et déconstruction », il a également été question de relecture et de réinterprétation des sources. Cela a conduit à reconsidérer l'ensemble du règne, sa chronologie mais aussi ses acteurs. Une des tendances des dernières années est de s'intéresser aux réseaux, aux groupements et à leurs interactions ; aussi des chercheurs ontils présenté leurs études sur la dynamique des pouvoirs locaux et la relation de l'empire carolingien avec les autres espaces. Le succès de ce colloque est dû à la pluridisciplinarité qui l'a caractérisé : historiens, historiens de l'art et archéologues ont pu échanger sur les dernières découvertes relatives au règne.

L'Institut historique allemand de Paris a aussi consacré deux tables rondes à Charlemagne. La première, organisée en avril 2013 et dont les actes ont été publiés en janvier 2016, s'est intéressée à un phénomène architectural récurrent de la période carolingienne : la multiplication des sanctuaires dans un même groupe cathédral ou monastique ${ }^{26}$. Le règne de Charlemagne a été particulièrement prospère en matière de fondations et de constructions de monastères et de cathédrales : il convenait donc de s'interroger sur les origines de cette profusion. L'intérêt de cette table ronde, intitulée «Groupes cathédraux et complexes monastiques : le phénomène de la pluralité des sanctuaires à l'époque carolingienne », était alors de remettre en question la part de nouveautés longtemps attribuée au seul règne de Charlemagne et de discuter des éventuels emprunts aux productions mérovingiennes, voire paléochrétiennes. Cette table ronde a elle aussi joué la carte de la pluridisciplinarité en convoquant historiens, historiens de l'art et archéologues pour débattre sur les diverses topographies, fonctionnalités et liturgies qui ont pu orienter les motivations des constructeurs vers ce type de monastères et de cathédrales à églises multiples ${ }^{27}$.

La seconde table ronde organisée en novembre 2013, et dont les actes sont en cours de publication, s'est concentrée sur les différents groupes sociaux qui cohabitaient à l'époque carolingienne (et plus particulièrement sous le règne de Charlemagne) et qui se distinguaient par leurs origines ethniques, culturelles et religieuses. La nouveauté de cette rencontre a été de s'intéresser non seulement à la perception qu'avait le pouvoir de ces minorités, par exemple les Juifs ou les Hispaniques, mais

26. M.-L. PAIN éd., Groupes cathédraux et complexes monastiques. Le phénomène de la pluralité des sanctuaires à l'époque carolingienne, Rennes, 2016.

27. Groupes cathédraux et complexes monastiques : le phénomène de la pluralité des sanctuaires à l'époque carolingienne, compte rendu (en allemand) de la table ronde par M.-L. PAIN, paru en ligne le 19 avril 2013 (http://www.hsozkult.de/conferencereport/id/ tagungsberichte-4894). 
aussi de comprendre les différents points de vue qu'avaient ces derniers sur les royaumes occidentaux. Une large diversité de sources a ainsi été exploitée, des chroniques aux actes juridiques en passant par les épitaphes. Les domaines abordés ont été variés puisqu'il s'agissait d'une étude qui devait être à la fois économique, politique, sociale, culturelle et religieuse. Ce qu'il en est ressorti est cette particulière complexité qui définit la période carolingienne et sa société ${ }^{28}$.

La Société internationale des médiévistes (International Medieval Society, Paris) a cherché à marquer l'anniversaire de la mort de Charlemagne par l'organisation d'un colloque aux ambitions novatrices. Charlemagne a été l'un des souverains les plus marquants de notre histoire, si bien que, dès la période médiévale, les sociétés en ont fait un modèle, un personnage littéraire et un saint. L'idée maîtresse était ainsi de réévaluer l'héritage laissé par Charlemagne dans les sociétés médiévales, de 814 jusqu'à la fin du Moyen Âge. La liturgie et les discours hagiographiques associés à ce personnage sanctifié ont fait l'objet de nouvelles études. De là, la représentation du souverain et le culte de son image ont été rediscutés à la lumière des derniers acquis de la recherche. Outre ces représentations artistiques, il a également été question des représentations littéraires à travers l'analyse de poésies, d'écrits historiques et de chansons de geste. Un des points intéressants soulevés lors de ce symposium a été le souvenir laissé par le souverain en tant que fondateur de monastères, de villes ou encore d'universités (au XVII ${ }^{\mathrm{e}}-\mathrm{XVIII}{ }^{\mathrm{e}}$ siècle, l'Université de Paris prétendait être une fondation de Charlemagne, et le séminaire de Zurich faisait de même). In fine, il convenait de redéfinir la figure légendaire de Charlemagne qui, depuis, a servi de nombreuses fois de modèle aux sociétés même contemporaines $^{29}$.

À Herstal, ville connue pour son célèbre capitulaire, une rencontre intitulée «Les Carolingiens dans le bassin mosan. Autour des palais de Herstal et de Jupille » a permis de croiser, dans un savant mélange pluridisciplinaire, les derniers résultats des recherches tant en histoire qu'en archéologie. Évidemment, c'est d'abord le capitulaire de 779 qui a été l'objet de toutes les attentions. Ce document est de première importance car il est le témoin le plus ancien des décisions collégiales prises par le roi et les grands du royaume - laïcs comme ecclésiastiques. À partir de cette date, Charlemagne entama une série de réformes considérée comme un tournant dans sa politique et sa gestion du royaume. Dans un second

28. Les altérités ethniques, culturelles et religieuses à l'époque carolingienne, compte rendu (en allemand) de la table ronde par A. SAGASSER, paru en ligne le 15 novembre 2013, http://www.hsozkult.de/conferencereport/id/tagungsberichte-5236.

29. Charlemagne after Charlemagne. 11. Jahrestagung der International Medieval Society, Paris, 26-28 juin 2014, compte rendu (en allemand) du colloque par J. FÜHRER, paru en ligne le 28 juin 2014 (http://www.hsozkult.de/conferencereport/id/tagungsberichte-5494). 
temps, l'actualité de la recherche archéologique a été mise à l'honneur avec un bilan des découvertes sur les sites de Jupille, Herstal, Huy, Villers-leBouillet et Engis. Outre les palais royaux, ce sont aussi des occupations modestes qui ont été étudiées afin de rendre compte, autant que possible, du mode de vie à l'époque carolingienne, que ce soit dans les hautes sphères de la société comme dans les zones rurales. Ce colloque a été accompagné d'une exposition qui présentait le personnage de Charlemagne, sa dynastie et la société franque du VIII ${ }^{\mathrm{e}}-\mathrm{IX}^{\mathrm{e}}$ siècle.

\section{Expositions et émissions en Europe}

Ce mille deux centième anniversaire de la mort de Charlemagne a également été l'occasion de présenter au public de riches expositions sur le règne du monarque. Celles-ci ont donné lieu à d'importantes publications comme à Zurich, en 2013, pour l'exposition «Karl der Grosse in der Schweiz $^{30} \gg$. L'ouvrage qui en a été tiré est essentiellement centré sur la production artistique à l'époque carolingienne - après avoir abordé quelques points historiques sur le lien entre Charlemagne et le territoire suisse. Ce dernier élément se révèle, par ailleurs, problématique : la Suisse en tant que telle n'est pas un territoire politiquement défini pour la période carolingienne. Si restreindre cette étude au seul territoire de la Suisse peut paraître anachronique d'un point de vue politique, on observe en revanche une même tendance artistique présentant des influences communes, franques et lombardes notamment. Aussi, cette publication est plutôt un panorama de ce qui s'est fait tant en architecture qu'en peinture, en sculpture et en orfèvrerie : soit un véritable inventaire des œuvres exécutées dans cette région. À travers différentes études de cas, l'ensemble des auteurs présente une tendance artistique commune à la Suisse malgré le brassage des influences, ce qui permet de mieux appréhender ce territoire pour l'époque concernée. Cette focalisation audacieuse paraît ainsi plus intéressante du point de vue de l'art que de la politique de Charlemagne. Elle est également l'occasion d'aborder l'actualité de la recherche en Suisse tant en histoire de l'art qu'en archéologie.

Mais l'événement attendu de cette année 2014 a été l'exposition organisée à Aix-la-Chapelle, « Karl der Grosse : Macht, Kunst und Schätze ». Cette importante rétrospective sur le règne de Charlemagne a donné lieu à la publication d'un catalogue en trois volumes ${ }^{31}$, qui présente non

30. G. Desceeudres, J. Goll et M. RieK éd., Die Zeit Karls des Grossen in der Schweiz, Sulgen, 2013.

31. F. Pohle, P. van DEN BRINKet al. éd., Karl der Große - Charlemagne, 3 vol., Dresde, 2014. 
seulement l'art à l'époque du monarque, mais aussi sa politique et l'étendue de sa domination - des thèmes abordés bien sûr depuis longtemps. Le premier volume traite de l'art carolingien en général, à travers les célèbres manuscrits qui ont fait la renommée des écoles du palais de Charlemagne en passant par l'orfèvrerie et l'architecture de la chapelle d'Aix. Si, dans le second volume, les sujets sur le pouvoir et la politique militaire de Charlemagne ont déjà été traités ailleurs, en revanche, le thème « mobilité et communication » est, on l'a vu, à l'honneur depuis peu dans les colloques et inscrit l'exposition dans cette nouvelle tendance historiographique. Dans cette partie de l'exposition, une grande diversité d'objets de voyage a été présentée : étrier, ampoule de pèlerin, bateau mais aussi cadeaux d'ambassade. Les ressources exploitées à l'époque carolingienne sont aussi étudiées attentivement, notamment à travers le capitulaire $D e$ villis. Le catalogue reprend l'éclectisme qui caractérisait l'exposition en publiant des notices sur des éléments de mobilier archéologique, en lien avec l'artisanat ou l'agriculture et tout ce qui était représentatif de la culture carolingienne et de sa société. Une large part a été accordée à l'étude de l'architecture et de sa valeur représentative du pouvoir, qu'il soit carolingien ou non. Ainsi, une grande partie des articles du catalogue se sont concentrés, à l'image de l'exposition, sur la ville d'Aix, son architecture, sa sculpture, l'état des fouilles archéologiques, mais aussi sur ses habitants et son aristocratie. Cet intérêt pour le pouvoir, la société et les réseaux a cependant laissé de côté l'Église, pourtant fermement liée à Charlemagne, à sa société et à son gouvernement. Le dernier volume du catalogue développe les axes majeurs de l'exposition à travers plusieurs articles, plus complets et plus exhaustifs. Ils présentent ainsi l'actualité de la recherche et orientent leurs propos vers les thèmes précédemment évoqués que sont les communications et les réseaux, les ressources, l'architecture et le pouvoir, ou encore le mythe de Charlemagne - thèmes à l'honneur depuis quelques années.

À Saint-Riquier dans la Somme, une exposition sur les Carolingiens - «L'Europe avant l'Europe. Les Carolingiens »- a été inaugurée à l'été 2014. Cette exposition assez hétéroclite, qui s'adressait à un vaste public, présentait, après une brève introduction sur l'abbaye, la société du haut Moyen Âge, de l'alimentation aux méthodes de chasse en passant par les manuscrits carolingiens et les Vikings. Il convient aussi de mentionner le projet d'exposition au Deutsches Historisches Museum de Berlin qui, finalement, n'a pas abouti. Il aurait été question d'un rapprochement entre l'empire carolingien, l'empire byzantin et l'empire abbasside. Un catalogue de cette exposition avortée est néanmoins paru ${ }^{32}$.

32. Deutsches Historisches Museum, Kaiser und Kalifen. Karl der Große und die Mächte am Mittelmeer um 800, Mayence, 2014. 
Nous pouvons achever ce tour d'horizon en évoquant l'intérêt qu'a suscité cette célébration dans d'autres media tels que la télévision. Le 20 avril 2013, la chaîne franco-allemande Arte a diffusé un documentairefiction sur Charlemagne ${ }^{33}$. Cette rétrospective sur son règne a pu ainsi être offerte à un large public. Alors que France Culture a consacré un feuilleton en quatre volets à Charlemagne dans l'émission La Fabrique de l'histoire ${ }^{34}$, la revue L'Histoire a publié un numéro spécial, où les transformations du Charlemagne historique en un personnage quasi mythique prennent une place importante ${ }^{35}$. Enfin, une bande dessinée retraçant l'histoire de Charlemagne depuis son avènement jusqu'à son couronnement impérial est parue au cours de cette même année 2014 chez Glénat : le travail des scénaristes a bénéficié des conseils de Geneviève Bührer-Thierry ${ }^{36}$.

\section{Au-delà des historiographies nationales : empire carolingien- empire chrétien}

Les travaux parus ces dernières années s'intéressent beaucoup à l'organisation interne de l'empire de Charlemagne. Cependant, cet empire n'était concevable que sous la forme d'un empire chrétien. Par conséquent il fallait, d'un côté, christianiser les régions de l'empire qui n'étaient pas, ou pas encore, chrétiennes, notamment la Saxe et, de l'autre, se mettre d'accord sur la vraie foi et propager celle-ci. À propos de la christianisation, de nouvelles recherches ont mis l'accent sur le fait que l'empire carolingien était en contact non seulement avec l'empire byzantin et le pape, mais aussi avec les puissances musulmanes de la péninsule Ibérique et du ProcheOrient où la chrétienté trouvait ses limites, même si l'Évangile selon saint Matthieu enjoignait les disciples de Jésus de propager la foi jusqu'aux extrémités du monde ${ }^{37}$. Pour la Saxe, la christianisation était imposée d'en haut, cela est bien connu, mais on a longtemps ignoré le caractère apparemment social de l'adhésion à la nouvelle religion ou de la réticence

33. Karl der Große - Charlemagne, documentaire de G. WENGLER, diffusé sur Arte le 20 avril 2013.

34. Charlemagne, émissions d'E. LAURENTIN, diffusées sur France Culture entre le 29 avril et le 2 mai 2013.

35. L'Histoire, $\mathrm{n}^{\circ} 406$ (Charlemagne : les habits neufs de l'empereur), décembre 2014.

36. Charlemagne, scénario C. BRUNEAU et V. DELMAS, dessin G. LEMERCIER, conseillère historique G. BÜHRER-THIERRY, Grenoble, 2014.

37. Mt 28,18-20. Voir L. E. VON PADBERG, Christianisierung im Mittelalter, Darmstadt, 2006, dont les arguments essentiels sont repris dans le cadre d'un catalogue d'exposition sur l'évangélisation à l'époque médiévale : ID., " Das Christentum als missionierende Religion. Missionskonzepte von Bonifatius bis ins späte Mittelalter », dans C. STIEGEMANN, M. KroKer et W. Walter éd., Credo. Christianisierung Europas im Mittelalter. Band 1 : Essays, Petersberg, 2013, p. 130-139. 
face à celle-ci. On croit savoir actuellement que les strates sociales les plus élevées des Saxons se sont ralliées plus rapidement à la domination franque et à la nouvelle religion, alors que des couches plus défavorisées, dont le statut allait encore s'altérer, restaient plus attachées au mode ancien ${ }^{38}$. Vers le milieu du $\mathrm{IX}^{\mathrm{e}}$ siècle, l'empereur Lothaire, voulant nuire à son frère cadet Louis le Germanique en charge de la Saxe, cherchait à se faire des alliés en Saxe en promettant aux Saxons qu'il leur laisserait le libre choix de leur religion - entendons par là qu'il leur aurait promis de les laisser retourner à leur ancienne religion, et cela quelque soixante-dix ans après le début de la conquête de la Saxe par Charlemagne.

Plus récemment encore, Carine van Rhijn a commencé à dépouiller un groupe de manuscrits qu'elle a pu identifier comme des manuels à l'usage du clergé local dans les provinces de l'empire carolingien ${ }^{39}$. Les études qui en découlent jettent quelque lumière sur la réalisation des réformes carolingiennes sur le terrain, et cela non seulement en Saxe, mais aussi en terre depuis longtemps chrétienne. On perçoit alors un désir de correctio et un sens de la précision pour le déroulement de la liturgie, des explications sur le sens du baptême, etc. avec, entre autres, le rappel qu'il faut nettoyer la vaisselle après avoir célébré l'eucharistie, éviter que les souris grignotent les hosties et veiller à chasser les chiens hors des églises. Dans la même optique, on peut citer les travaux de Susan Keefe ; à son grand travail en deux volumes ${ }^{40}$ s'ajoutent la publication d'un catalogue d'ouvrages de l'époque carolingienne qui traitent de la profession de la foi ${ }^{41}$, et une édition de textes de ce genre dans un volume du Corpus Christianorum $^{42}$. Elle prête une attention particulière au contexte dans lequel ces textes se trouvent insérés dans les manuscrits.

Les recherches menées par les précédentes générations ont montré que les réformes carolingiennes étaient au service d'une réforme de la liturgie. C'est dans ce sillon que s'inscrit un renouveau assez significatif des

38. M. LINTZEL, Der sächsische Stammesstaat und seine Eroberung durch die Franken, Berlin, 1933, repris dans W. LAMMERS éd., Entstehung und Verfassung des Sachsenstammes, Darmstadt, 1967 (Wege der Forschung, 50), p. 149-206.

39. Voir par exemple C. VANRHIJN, «Charlemagne's correctio : A Local Perspective ", communication présentée lors du colloque Charlemagne. Les temps, les espaces, les hommes. Construction et déconstruction d'un règne, Paris, 26 mars 2014 (actes du colloque en cours de préparation) ; EAD., « The Local Church, Priests' Handbooks and Pastoral Care in the Carolingian Period », dans E. MENESTÒ éd., Chiese locali e chiese regionali nell'alto medioevo, Spolète, 2014, p. 689-706.

40. S. KeEFE, Water and the Word. Baptism and the Instruction of the Clergy in the Carolingian Empire, 2 vol., Notre Dame, 2002.

41. S. KeEFE, A Catalogue of Works Pertaining to the Explanation of the Creed in Carolingian Manuscripts, Turnhout, 2012.

42. Explanationes Symboli aeui Carolini, éd. S. KeEFE, Turnhout, 2012 (Corpus Christianorum, Continuatio Mediaevalis, 254). 
recherches dans le domaine de l'histoire de la chrétienté et de la théologie liée à l'histoire politique entendue au sens large du terme. Ainsi, Florence Close, dans son livre substantiel, Uniformiser la foi pour unifier l'Empire. Contributionà l'histoire de la pensée politico-théologique de Charlemagne ${ }^{43}$, choisit l'empire carolingien comme point de départ pour un examen des débats trinitaires dont on relève les traces aussi bien dans les diplômes de Charlemagne que dans les conciles de cette époque et notamment dans les écrits d'Alcuin. Selon Florence Close, le programme de réforme carolingien était régi par Alcuin, fortement intéressé par les questions liées au dogme trinitaire, d'autant plus que ce dogme était au centre de débats théologiques qui trouvèrent, par exemple, leur expression au concile de Francfort de 794. Si l'unité de la foi n'avait pas été imposée partout dans l'empire, l'unité politique n'aurait plus été assurée. Finalement, les questions du baptême avaient une telle importance qu'Owen M. Phelan, auteur d'un livre sur la théologie sacramentelle du baptême à l'époque carolingienne, n'hésite pas à intituler son ouvrage The Formation of Christian Europe ${ }^{44}$. Citons la première phrase du livre afin de montrer à quel point cette question est considérée comme fondamentale ${ }^{45}$ :

Around the turn of the ninth century, political and religious reformers worked to establish an imperium christianum - a Christian empire - a society whose most basic organizing principle was the sacramentum of baptism.

Il est effectivement vrai que le travail d'uniformisation au sein de l'empire carolingien passait visiblement par une réflexion sur les moyens qui pourraient conduire à l'unité, ce qui signifie qu'il fallait également réfléchir à la manière dont ce message allait arriver dans chaque village. Il fallait alors passer par le réseau de l'Église. Peut-être les sources subsistantes nous présentent-elles une image un peu trop axée sur le clergé, du fait de la transmission cléricale des textes. Mais c'est là encore un autre sujet à propos duquel les recherches sont en cours et promettent des progrès notables.

\section{Charlemagne au XXI ${ }^{\mathrm{e}}$ siècle}

Nous espérons avoir pu montrer que la recherche sur l'empire à l'époque carolingienne a connu un renouveau surprenant lors des dernières années. Si le sujet a longtemps été délaissé par la communauté scientifique, de nouvelles parutions en français, mais aussi en anglais et en allemand,

43. F. Close, Uniformiser la foi pour unifier l'Empire. Contribution à l'histoire de la pensée politico-théologique de Charlemagne, Bruxelles, 2011.

44. O. M. Phelan, The Formation of Christian Europe. The Carolingians, Baptism, and the Imperium Christianum, Oxford, 2014.

45. Ibid., p. 1. 
ont défriché de nouvelles voies et posé de nouvelles questions, de sorte que même des biographies d'apparence classique peuvent contenir des mises en perspective assez inédites. Plusieurs projets d'éditions critiques sont en cours d'élaboration, car, si la Vie de Charlemagne d'Éginhard est parue dans une nouvelle édition avec traduction et notes, on attend encore de nouvelles éditions des capitulaires, des lettres de Loup de Ferrières, des lettres d'Éginhard, des Annales de Saint-Gall, etc., dont la réalisation a été commencée, qui changeront à nouveau nos perspectives et qui permettront sans doute d'en finir avec quelques idées reçues provenant souvent d'éditions anciennes et de traductions fautives. Il y aurait aussi beaucoup à attendre de nouvelles éditions des lois « barbares $^{46}$ », mais pour l'instant, aucun projet n'est en route. Nous n'avons pas mentionné les travaux de sémantique historique paraissant actuellement, surtout en Allemagne ${ }^{47}$; on pourrait également évoquer les études comparatistes citées qui, influencées par la mondialisation qui touche également les études médiévales, essayent de rapprocher les structures administratives, par exemple, de l'empire carolingien, de l'empire byzantin et de la Chine ${ }^{48}$, mais cela nous éloignerait de notre propos. Les termes d' « organisation de l'empire » et d' " empire chrétien » constituent donc les centres d'intérêt qui représentent le mieux l'actualité historiographique de l'empire carolingien.

\author{
Julian Führer - Historisches Seminar (Zürich) \\ Marie-Laure Pain - Deutsches Historisches Institut (Paris) \\ Amélie Sagasser - Hochschule für jüdische Studien (Heidelberg)
}

\title{
Charlemagne, 814-2014 : bilan historiographique d'une année de commémoration
}

Cette étude se propose de dresser un panorama de la production historiographique de "l'année Charlemagne » 2014, mille deux cents ans après la mort du souverain. Sans prétendre à l'exhaustivité, les auteurs inscrivent les publications récentes dans le cadre des traditions historiographiques du $X I X^{e}-X X^{e}$ siècle des deuX côtés du Rhin. Sont ensuite passées en revue les diverses manifestations scientifiques, expositions et parutions qui ont marqué cette année. Cet examen a révélé deux tendances : plusieurs nouvelles biographies ont vu le jour en Allemagne, alors qu'en France, le personnage a été commémoré différemment. Un courant historiographique très actif dans plusieurs pays s'intéresse à la création de l'empire uni dans la foi chrétienne et à la création et l'implantation de cette foi, à travers des réflexions théologiques mises en application sur le terrain.

Charlemagne - empire - haut Moyen Âge - historiographie - théologie

46. T. LIENHARD, « L'historiographie germanophone sur les lois barbares : centres de gravité, évolutions, desiderata », Revue de l'Institut français d'histoire en Allemagne, 2 (2010), p. 133-163.

47. Par exemple, pour un terme clé de l'histoire de l'empire carolingien, T. GEelHAAR, Christianitas. Eine Wortgeschichte von der Spätantike bis zum Mittelalter, Göttingen, 2015.

48. M. BORGOLTE, « Karl der Große... ». 
Charlemagne, 814-2014 : Historiographical Report on a Year of Commemoration

The article wants to offer a survey of the new historiographical production of the "Charlemagne Year " 2014, twelve centuries after the emperor's death. It doesn't pretend to be exhaustive. The authors embed the new publications into the historiographical traditions of both Germany and France in the nineteenth and twentieth centuries. Then, the article examines the scholarly events, exhibitions and new publications of that year. It appears that a couple of new biographies has been published in German and that, in France, Charlemagne has been commemorated in a different way. A new and very active historiographical stream examines the creation of a Christian empire based on the Christian faith and the implementation of this faith, with a special accent on theological reflections and their application.

Charlemagne - Historiography - Empire - Early Middle Ages - Theology 
